Article

\title{
Electrical Detection of Creeping Discharges over Insulator Surfaces in Atmospheric Gases Under AC Voltage Application
}

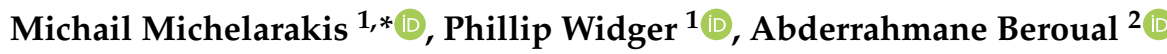 \\ and Abderrahmane (Manu) Haddad ${ }^{1}$ (D) \\ 1 Advanced High Voltage Engineering Research Centre, School of Engineering, Cardiff University, The Parade, \\ Cardiff CF24 3AA, UK \\ 2 École Centrale de Lyon, University of Lyon, Ampère CNRS UMR 5005, 36 Avenue Guy Collongue, \\ 69134 Écully, France \\ * Correspondence: MichelarakisM@cardiff.ac.uk
}

Received: 30 June 2019; Accepted: 30 July 2019; Published: 1 August 2019

\begin{abstract}
Creeping discharges over insulator surfaces have been related to the presence of triple junctions in compressed gas insulated systems. The performance of dielectric materials frequently utilised in gaseous insulating high voltage applications, stressed under triple junction conditions, has been an interesting topic approached through many different physical perspectives. Presented research outcomes have contributed to the understanding of the mechanisms behind the related phenomena, macroscopically and microscopically. This paper deals with the electrical detection of creeping discharges over disc-shaped insulator samples of different dielectric materials (polytetrafluoroethylene (PTFE), epoxy resin and silicone rubber) using atmospheric gases (dry air, $\mathrm{N}_{2}$ and $\mathrm{CO}_{2}$ ) as insulation medium in a point-plane electrode arrangement and under AC voltage application. The entire approach implementation is described in detail, from the initial numerical field simulations of the electrode configuration to the sensing and recording devices specifications and applications. The obtained results demonstrate the dependence of the generated discharge activity on the geometrical and material properties of the dielectric and the solid/atmospheric gas interface. The current work will be further extended as part of a future extensive research programme.
\end{abstract}

Keywords: creeping discharge; AC voltage; point-plane; atmospheric gases; flashover voltage; polytetrafluoroethylene (PTFE); epoxy resin; silicone rubber

\section{Introduction}

In gaseous insulation applications, triple junctions are defined as points where the gaseous medium, dielectric material and electrode meet leading to local electric field enhancements. When a certain electric field level is reached, the initiation of discharge activity is possible. In the case of their appearance, such phenomena become present at voltages below the optimised rated operating and withstand levels of the affected apparatus, introducing additional concerns in the overall effort of designing effective and reliable insulating systems. Some significant examples of triple junctions are incorporated into the design of Gas Insulated Lines (GIL), Gas Insulating Switchgear (GIS), transformer bushings and Gas Circuit Breakers (GCB).

The most common discharge phenomenon linked with enhanced electric fields in the vicinity of solid dielectric-gas interface is the streamer creeping discharge. Over the years, major contributions have been published, approaching the development and propagation of streamers over dielectric surfaces from several different experimental and computational perspectives. Characteristics of single streamer events in homogenous electric field arrangements have been reported [1,2] where the 
dependence of the propagation velocity and associated electric field over the dielectric material is described. In [3], the effect of the dielectric permittivity on streamer propagation along insulating surfaces using electrical and optical techniques is reported. The morphology and propagation length of creeping discharges and their dependence on voltage waveform, voltage levels, dielectric material and gaseous medium have been extensively studied in more recent works [4-7]. Reported works using dust figure [8] and Pockels effect methods [9] always constitute very interesting optical approaches. Another very important aspect, related to the phenomena described above, is the surface charge accumulation and its impact on the flashover voltage $[10,11]$ and degradation of solid insulators under tests [12].

This paper examines the development of creeping discharge over disc-shaped insulator samples of different dielectric materials, namely polytetrafluoroethylene (PTFE), epoxy resin and silicone rubber, which are frequently utilised in high voltage technology applications. These samples are also insulated by a gaseous medium of either dry air, nitrogen $\left(\mathrm{N}_{2}\right)$ or carbon dioxide $\left(\mathrm{CO}_{2}\right)$. A needle-plane electrode configuration is employed with a strongly non-uniform electric field at $50 \mathrm{~Hz} \mathrm{AC}$ voltage applied to the needle electrode, in order to replicate triple junction conditions. The implementation of the test procedure is described from the early stages of the numerical field simulation process of the electrodes arrangement using a Finite Element Solver (FEM) software package. The aim of the accurate modeling of the electrode geometry is the optimisation of the electric field distribution along the insulator surface. Detection of surface discharges is performed by means of sensing associated currents using a high-sensitivity, high frequency current transformer (HFCT) ranging from a few $\mathrm{kHz}$ up to several $\mathrm{MHz}$ bandwidth. The current transformer sensing technique provides several performance advantages over other techniques implementing different physical principles [13], such as low power loss, high accuracy and no need for further amplification of the output. Additionally, the overall convenience of a HFCT installation, together with the provided electrical isolation between the system under test and the high-cost recording devices, make the technique a very practical choice for laboratory-based high voltage testing.

\section{Design and Implementation of the Test Procedure}

\subsection{Electrodes Configuration Simulation and Electric Field Computation}

As mentioned in the previous section, the aim of the test procedure is to replicate triple junction conditions in the vicinity of a dielectric insulator surface surrounded by a gaseous insulating medium. For that reason, a needle-plane electrode configuration is employed. A two-dimensional illustration along with an actual picture of the electrode configuration, showing the disc-shaped insulator sample under test conditions are shown in Figure 1.

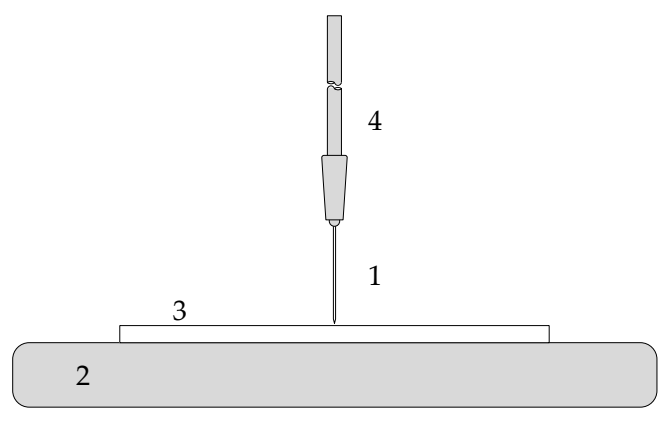

(a)

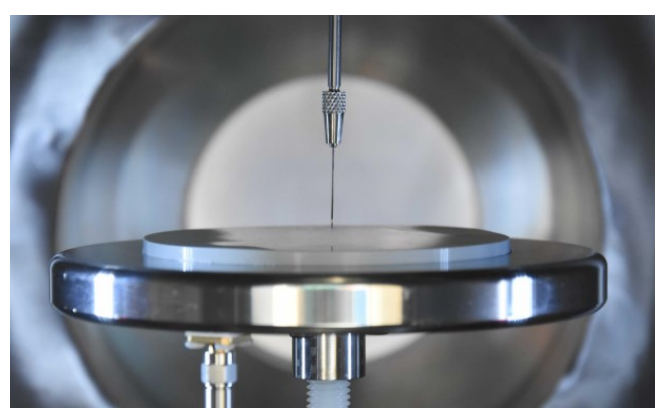

(b)

Figure 1. Needle-plane electrode configuration with a disc-shaped insulator sample: (a) Two-dimensional illustration of the configuration with the following numbered features: 1-needle electrode, 2-plane electrode, 3-disc-shaped insulator sample and 4-needle holder; (b) Actual picture of the practical test configuration as set-up inside the pressure vessel. 
The needle is made of tungsten with a high-precision tip diameter of $20 \pm 0.5 \mu \mathrm{m}, 0.51 \mathrm{~mm}$ shaft diameter and a length of $32 \mathrm{~mm}$. It is attached to a stainless-steel needle holder placed perpendicularly and in close proximity to the insulator sample disc center. The plane electrode incorporates a dull polished stainless-steel planar surface of $150 \mathrm{~mm}$ diameter and $15 \mathrm{~mm}$ thickness and is electrically separated from the rest of the mounting system with a nylon threaded rod. The entire configuration is placed vertically and centered inside a cylindrical 901 dull polished stainless-steel pressure vessel rated up to 10 bars operational gauge pressure, which has a diameter of $480 \mathrm{~mm}$ and a height of $500 \mathrm{~mm}$.

The described design is further examined from the numerical electric field perspective using a Finite Element Method (FEM) simulation model. The detailed geometry is transferred to COMSOL Multiphysics ${ }^{\circledR}$ through its integrated three-dimensional geometry builder, with some geometry simplifications that can improve significantly the computational time without affecting the quality and reliability of the generated result. As the design is largely symmetrical around the central axis of the entire configuration, only half of the geometry needs to be computationally solved without affecting the accuracy of the computed results. It is expected that the maximum electric field stress will appear at the needle tip because of its small diameter and short distance from the zero potential plane electrode. Figure 2 shows the simulated full geometry of the test system, as designed within COMSOL Multiphysics ${ }^{\circledR}$, together with a generated illustration of the three-dimensional equipotential surfaces (isosurfaces) after solving the simulation model. For the example shown in Figure 2b, the considered insulator sample is made of PTFE with a $4 \mathrm{~mm}$ thickness; the insulating gaseous medium being air at standard atmospheric conditions. The applied voltage is set to $1 \mathrm{~V}$.

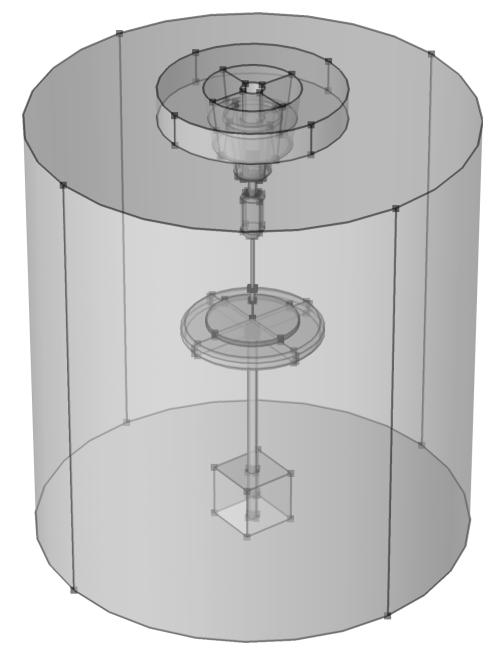

(a)

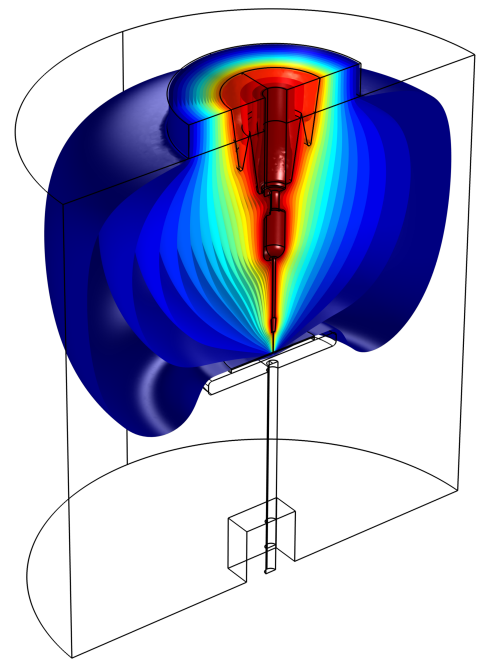

(b)

Figure 2. Finite Element Method (FEM) simulation model: (a) Full geometry including electrodes arrangement and surrounding volume; (b) Three-dimensional equipotential surfaces (isosurfaces) within the half symmetrical volume of the initial geometry.

The distribution of the electric field on the surface of the insulator sample is of great significance as the possibility of large irregularities may lead to inaccurate observations. In a broad sense, in case of discharge activity appearance, it will initiate from the sharp needle tip and will have equal chance of propagating in any direction towards the edges of the disc insulator. Figure 3 shows the computed electric field distribution along the diameter of the insulator sample top surface. The presented electric field values are normalised to the maximum surface electric field that appears at the point with the shortest distance from the needle tip. 


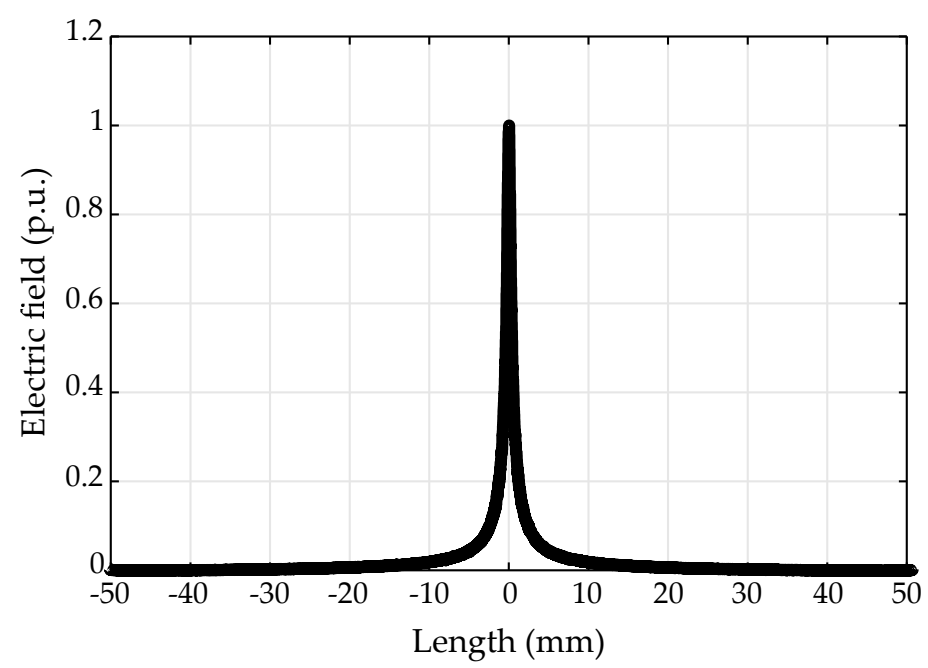

Figure 3. Electric field distribution along insulator surface. Here, $0 \mathrm{~mm}$ represents the center of the top surface of the insulator sample while $\pm 50 \mathrm{~mm}$ the edges of it. The presented values are normalised based on the maximum computed value.

\subsection{Experimental Set-Up}

Figure 4 summarises the main parts of the experimental configuration used for the purposes of this work. For the AC voltage application, a $50 \mathrm{kV} / 3.75 \mathrm{kVA}$ transformer is used, which is controlled through an isolating transformer and a voltage regulator with adjustment of the voltage level on the low-voltage side. An RC voltage divider of ratio 3750:1 is used to scale down the generated voltage to safe measurable levels. Voltage is applied to the pressure vessel through a bushing rated up to $39 \mathrm{kV}$ AC rms. As described previously, the electrodes configuration, together with the insulator samples under test, are vertically and horizontally centered inside the cylindrical stainless-steel dull polished chamber. The side apertures are covered with stainless-steel blanking plates, preventing ambient light entering into the pressure vessel.

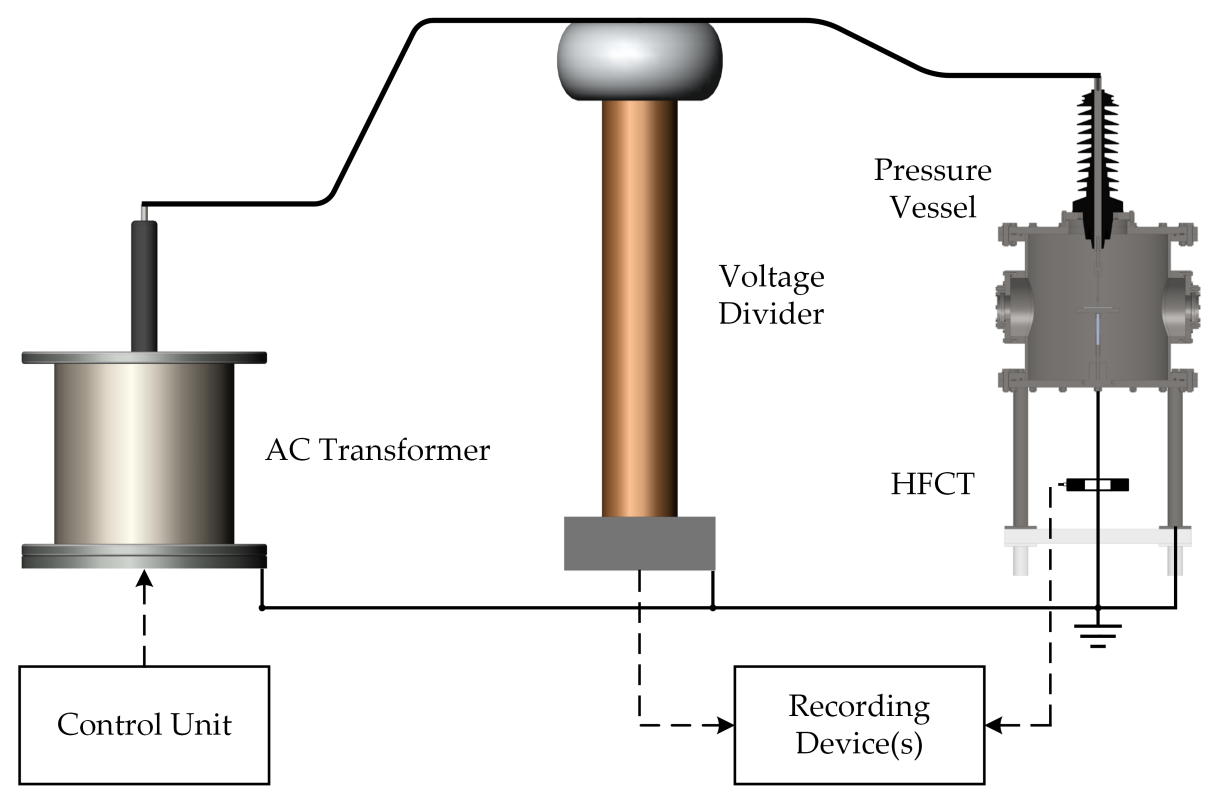

Figure 4. Experimental set-up for AC voltage application to the needle-plane configuration and insulator samples. 
The insulator samples tested are disc-shaped with a diameter of $100 \mathrm{~mm}$ and thicknesses of 4 and $6 \mathrm{~mm}$. Three different materials are considered namely polytetrafluoroethylene (PTFE), silicone rubber and epoxy resin with relative permittivities $\left(\varepsilon_{\mathrm{r}}\right)$ of 2.1, 2.9 and 3.5, respectively. The PTFE and epoxy resin samples surfaces were polished with sandpaper of 800 grit gradually increasing up to 7000 grit size. The silicone rubber samples were vacuum casted using a highly polished stainless-steel mold, followed by at least $24 \mathrm{~h}$ curing at $50{ }^{\circ} \mathrm{C}$. For all cases, arithmetical mean height $\left(R_{\mathrm{a}}\right)$ is maintained between $0.6-0.8 \mu \mathrm{m}$. The insulator samples are cleaned with high-purity isopropyl alcohol and dried for at least $4 \mathrm{~h}$ at $50{ }^{\circ} \mathrm{C}$. Between drying and placing the samples inside the final test set-up, a brief time interval of $30 \mathrm{~min}$ is kept allowing the samples to return to room temperature levels. After each test series, each insulator is examined for traces, indicating degradation/damage, and if any is observed on the surface, it is replaced with a new unused one. As described previously, the needles are made of pure tungsten, a material well-known for its high melting temperature, allowing for a sufficiently large number of tests without degradation of the needle tip quality. During the creeping discharge detection tests, a maximum of twenty voltage applications are made before the needle is replaced. For the case of flashover tests, the needle is used for a maximum of ten attempts prior to being replaced, assuming that no abrupt deviations in the readings of successive attempts occur which would indicate a damaged needle. For the purposes of the presented work, the test vessel housing the test electrode configuration is filled and tested using three different atmospheric gases, respectively: dry air, nitrogen $\left(\mathrm{N}_{2}\right)$ and carbon dioxide $\left(\mathrm{CO}_{2}\right)$ at 1 bar absolute pressure. Preceding gas injection, the pressure vessel is vacuumed for $30 \mathrm{~min}$ after a vacuum level of $-1000 \mathrm{mbar}$ gauge pressure is reached. This additional vacuum is held in an attempt to maintain low humidity levels inside the test chamber.

Two different, although similar, patterns for the AC voltage application are followed. For flashover tests, the applied voltage is manually increased with a rate of $1 \mathrm{kV} / \mathrm{s}$ until flashover occurs. A lower sensitivity, $0.1 \mathrm{~V} / \mathrm{A}$, current transformer is used for triggering the recording device. The last full AC-cycle recorded, preceding the flashover event, corresponds to the measured rms flashover voltage. Ten flashover tests are performed for each insulator sample/gaseous medium combination. The arithmetic mean value and standard deviation for the flashover voltage for each case are specified as implied from the relevant standards [14]. For creeping discharge detection tests, test voltages are specified as a percentage of the flashover voltage for each case study. Following that, the test voltage is gradually reached with an increment of $2 \%$ per second resulting in a ramp duration of $50 \mathrm{~s}$ for each case. After that, the voltage level is maintained for a maximum of $10 \mathrm{~s}$ in order to avoid overstress of the insulator sample and the utilised needle. Between two successive voltage applications, a time interval of at least $2 \mathrm{~min}$ is maintained.

\subsection{Current Sensing \& Recording}

Several methods have been reported for the detection of electrical phenomena within the high-frequency (HF), very-high-frequency (VHF) and ultra-high-frequency (UHF) regions, including acoustic, optical, chemical and electrical methods. These methods find wide application in the detection of partial discharges [15-19]. In this work, direct measurement of the currents associated with creeping discharge is performed using a high-sensitivity $5 \mathrm{~V} / \mathrm{A}$, high frequency current transformer (HFCT) ranging from $4.8 \mathrm{kHz}$ up to $400 \mathrm{MHz}$. Considering that the test configuration is installed inside a grounded metal fabricated pressure vessel, it is important that the generating signals will exit the test chamber and, consequently, reach the sensing device undistorted and unattenuated. For that purpose, a flange mount SMA connector is installed at the bottom side of the plane electrode of Figure 1. A $50 \Omega$ RG-405 coaxial cable connects the plane electrode with a pre-installed high-pressure rated coaxial feedthrough. A coaxial cable connected to the low-pressure side of the feedthrough is then connected to the configuration shown in Figure 5, similar implementations of which were presented in $[20,21]$. 


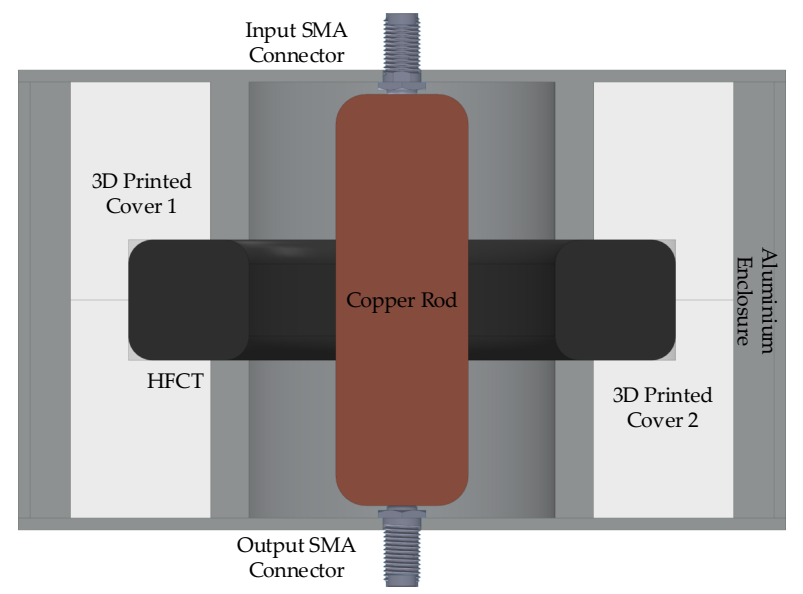

Figure 5. HFCT enclosure configuration.

Two SMA connectors are mounted on the top and bottom of a C101 alloy copper rod. The diameter of this central conductor was calculated based on the $50 \Omega$ coaxial characteristic impedance principle using Equation (1) [15],

$$
Z_{0}=\frac{138}{\sqrt{\varepsilon_{\text {air }}}} \log \left(\frac{d_{1}}{d_{2}}\right)
$$

where, $Z_{0}$ is the characteristic impedance, $d_{1}$ the diameter of the HFCT aperture, $d_{2}$ the diameter of the copper conductor and $\varepsilon_{\text {air }}$ the relative permittivity of atmospheric air. The entire configuration is enclosed in an EMI/RFI shielded aluminium enclosure. The scattering parameters of the configuration were measured using an $R \& S^{\circledR}$ ZVL Vector Network Analyzer $(9 \mathrm{kHz}-6 \mathrm{GHz})$, following a $50 \Omega$ two-port calibration, and the results are presented in Figure 6. As shown, the upper cut-off frequency is measured at $359.46 \mathrm{MHz}$, which is quite close to the bandwidth rating of the HFCT. The output side is terminated through a short-circuited $20 \mathrm{~dB} / 50 \mathrm{~W}, \mathrm{DC}-8.5 \mathrm{GHz}$, high-power fixed attenuator, preventing possible reflections reaching the sensing configuration.

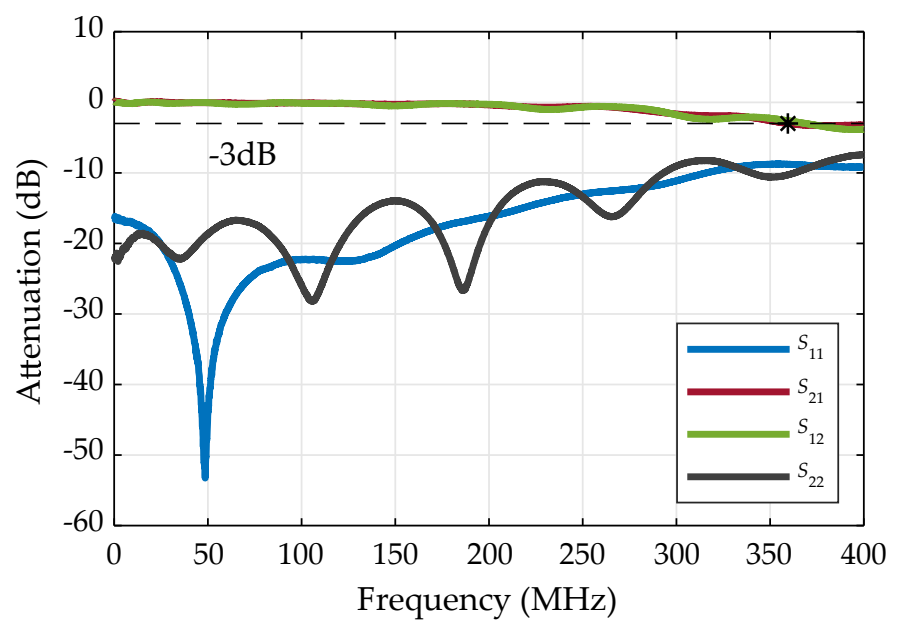

Figure 6. S-parameters of the configuration shown in Figure 5, for the frequency range corresponding to the rating of the used HFCT.

The recording of the voltage waveform and associated currents was performed in two different stages: high-resolution single trigger recordings were made using a Teledyne LeCroy HDO6104, $1 \mathrm{GHz}$, 2.5 GS/s, 12-bit oscilloscope while, for multiple successive trigger events, a large buffer PicoScope ${ }^{\circledR}$ 5000 series, $200 \mathrm{MHz}, 250 \mathrm{MS} / \mathrm{s}$, 12-bit was used. 


\section{Experimental Results and Discussion}

\subsection{Flashover Tests}

A series of flashover tests were performed using all the solid dielectric-insulating gas combinations considered in this work. Ten flashover events were recorded for each case. These tests were implemented in order to quantify the equivalent threshold voltage level for creeping discharges as a percentage of the corresponding mean flashover voltages of each case. The obtained results are summarised in Figure 7. For the case of the $6 \mathrm{~mm}$ thick PTFE sample in dry air, no flashover events were recorded within the allowed application voltage levels up to $39 \mathrm{kV} \mathrm{rms}$, hence, it is not included in the graph.

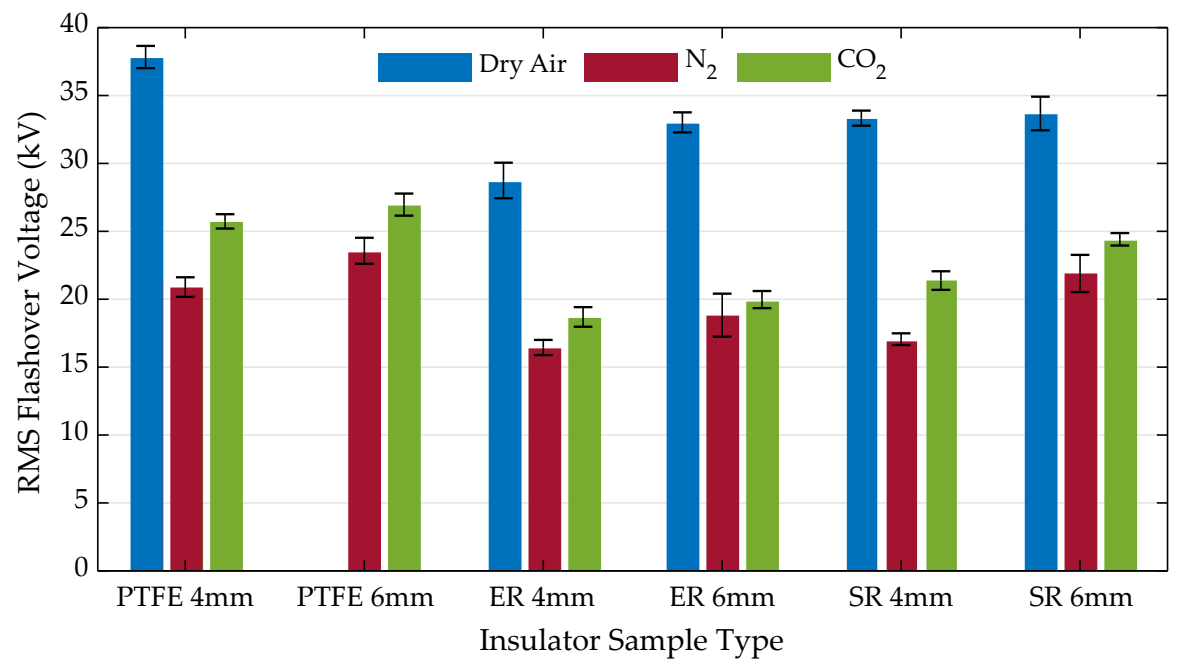

Figure 7. Flashover voltage (FOV) test results for six different insulator samples in dry air, nitrogen $\left(\mathrm{N}_{2}\right)$ and carbon dioxide $\left(\mathrm{CO}_{2}\right)$, all at 1 bar absolute pressure. Here, arithmetic mean values are depicted together with the corresponding standard deviations after 10 voltage applications. ER stands for epoxy resin while SR for silicone rubber.

For all the different sample types, dry air showed the best insulating performance as compared to the two other gaseous mediums. Additionally, pure $\mathrm{CO}_{2}$ showed a better performance compared to pure nitrogen, with the difference in FOVs being larger for $4 \mathrm{~mm}$ samples compared to those of $6 \mathrm{~mm}$ thickness. Focusing on the calculated standard deviations, $\mathrm{N}_{2}$ showed a more stable insulating behaviour for thinner samples, for all the materials compared to thicker ones insulated with the same gas, while $\mathrm{CO}_{2}$ seemed to have on average the lowest standard deviations. The electrode system with PTFE $\left(\varepsilon_{\mathrm{PTFE}}=2.1\right)$ had the highest resistance to flashover for all gaseous mediums and sample thicknesses considered. Despite the results of the other two materials being close, the configuration with silicone rubber $\left(\varepsilon_{\mathrm{SR}}=2.9\right)$ seemed to have a slightly higher withstand voltage compared to that with epoxy resin $\left(\varepsilon_{\mathrm{ER}}=3.5\right)$. Figure 8 shows the dependence of the computed electric field and measured flashover voltages on the different values of dielectric permittivity. The values of each curve are normalised to the, computed or measured, corresponding value for PTFE. It is obvious that, as the dielectric permittivity increases, the electric field on the center of the disc insulator sample also increases, while, the flashover voltage levels decrease for all the cases considered. Such an observation can be correlated with results reported in research works [5] where the stopping length of creeping discharges is examined, showing that insulator discs made of higher dielectric permittivity materials are responsible for the propagation of longer streamer channels on their surface for both $\mathrm{N}_{2}$ and $\mathrm{CO}_{2}$, but also for $\mathrm{SF}_{6}$. AC breakdown test results of point-plane arrangements for different gap distances, presented in [7], also showed that the insulating performance of $\mathrm{CO}_{2}$ is better when compared to $\mathrm{N}_{2}$, without a dielectric material between the electrodes. 


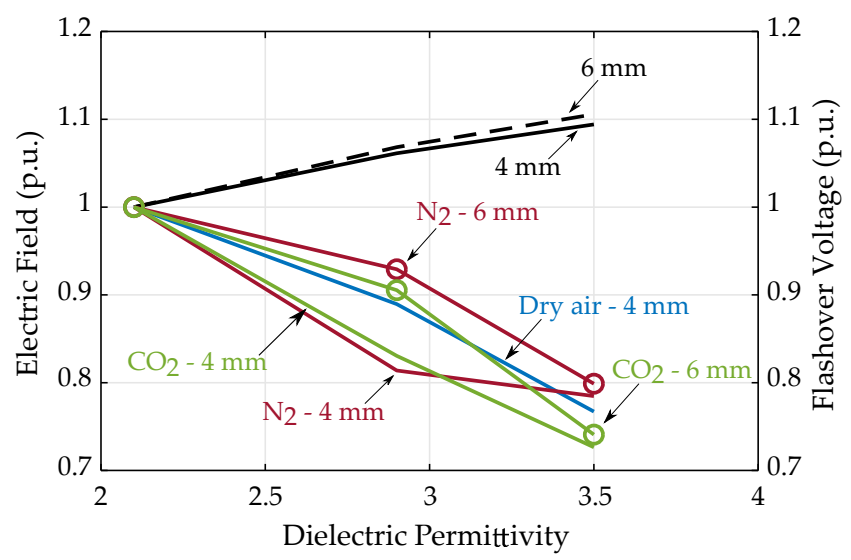

Figure 8. Computed electric field (black curves) and measured flashover voltages over dielectric permittivity variation. All the curves are normalised over the corresponding value of $\varepsilon_{\mathrm{r}}=2.1$ for PTFE.

\subsection{High-Resolution Recordings}

High-resolution recordings were obtained through single trigger events of the recording device at dual-channel, sampling at $1.25 \mathrm{GS} / \mathrm{s}$ rate. Two signals of $40 \mathrm{~ms}$ duration each were captured, corresponding to two full AC-cycles. The applied voltage levels considered for each case correspond to $85 \%$ of the flashover levels presented in previous section. That way, it is possible to compare between the different cases, where the variations in the FOVs do not allow the application of the same test voltage levels. Additionally, a 15\% safety margin from flashover events was maintained, avoiding unwanted stress of the test configuration in case of flashover. Figure 9 shows the obtained recordings for PTFE of $4 \mathrm{~mm}$ thickness in dry air, nitrogen $\left(\mathrm{N}_{2}\right)$ and carbon dioxide $\left(\mathrm{CO}_{2}\right)$ at 1 bar absolute pressure. Because similar patterns were observed for the remaining insulator samples types, only the above selected results were shown here.

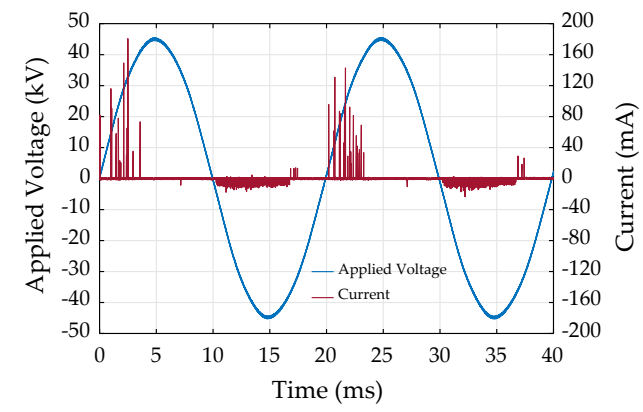

(a)

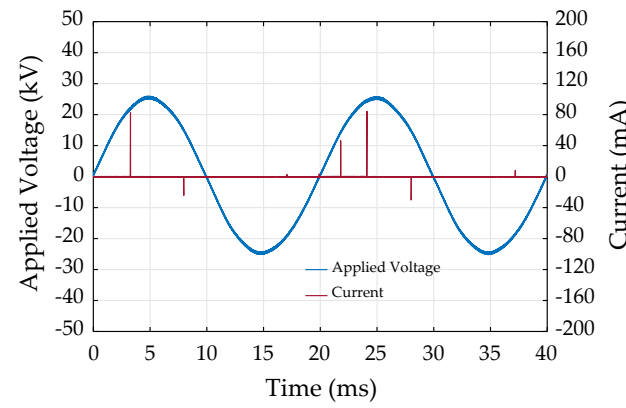

(b)

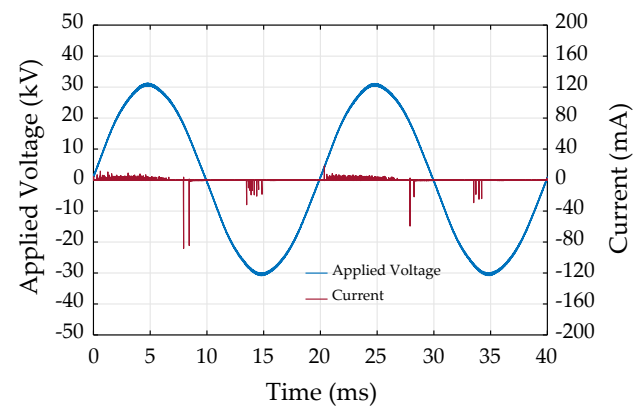

(c)

Figure 9. PTFE of $4 \mathrm{~mm}$ thickness in (a) dry air at 1 bar absolute pressure and $31.74 \mathrm{kV}$ rms applied voltage, (b) $\mathrm{N}_{2}$ at 1 bar absolute pressure and $17.74 \mathrm{kV} \mathrm{rms}$ applied voltage and (c) $\mathrm{CO}_{2}$ at 1 bar absolute pressure and $21.61 \mathrm{kV}$ rms applied voltage. 
For the case of dry air in Figure 9a, a high density of positive and negative polarity current pulses is observed. It is obvious that positive polarity current peaks amplitudes dominate over the negative spikes. However, significant differences in the current pulse characteristics were observed. For nitrogen $\left(\mathrm{N}_{2}\right)$, as shown in Figure $9 \mathrm{~b}$, a very different behaviour is observed when compared to dry air. During the positive AC half cycle of the applied voltage, a small number of positive current pulses, although quite high in amplitude, were detected while absence of activity during the negative half cycle is obvious. Finally in Figure $9 c$, the behaviour of carbon dioxide $\left(\mathrm{CO}_{2}\right)$ differs significantly from both dry air and $\mathrm{N}_{2}$. Low amplitude positive current pulses were detected during the positive half cycle of the applied voltage while, during the negative half cycle, higher amplitude but less dense negative polarity current pulses were observed.

Wide bandwidth sensing and recording devices combined with high sampling rates allow the capturing of current pulses corresponding to creeping discharge events. For the presented test results, applied voltages were very close to the FOVs and most of the recordings consist of superimposed pulses, indicating multiple discharge events occurring in very fast time frames on the surface of the insulator sample. Single streamer current pulses are also present however, less frequent. Current pulses are classified into two different categories: those having the same polarity as the applied voltage and those with opposite polarity. Positive polarity pulses during the positive half cycle of the AC voltage application are present for all the cases with varying amplitudes and characteristics. Examples of positive polarity pulses exported from the recordings in Figure 9 are shown in Figure 10 with their time resolved characteristics being summarised in Table 1. Negative polarity current pulses are also detected, the recordings of which are shown in Figure 11, with their corresponding characteristics summarised in Table 2. Similar pulse shapes were observed for all the insulator sample materials. The apparent charge, which is also depicted, was calculated by integrating the recorded pulse over the corresponding time domain.

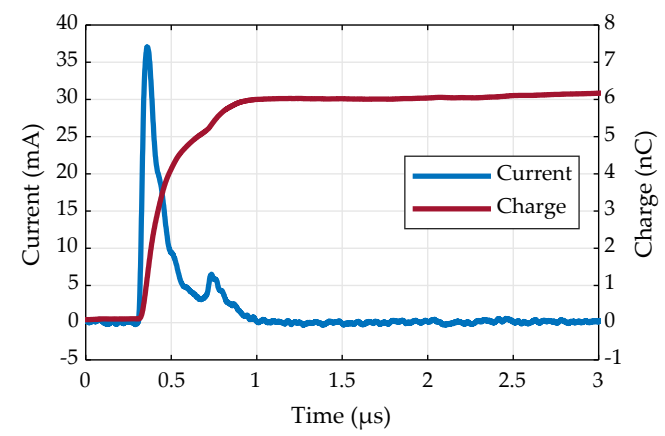

(a)

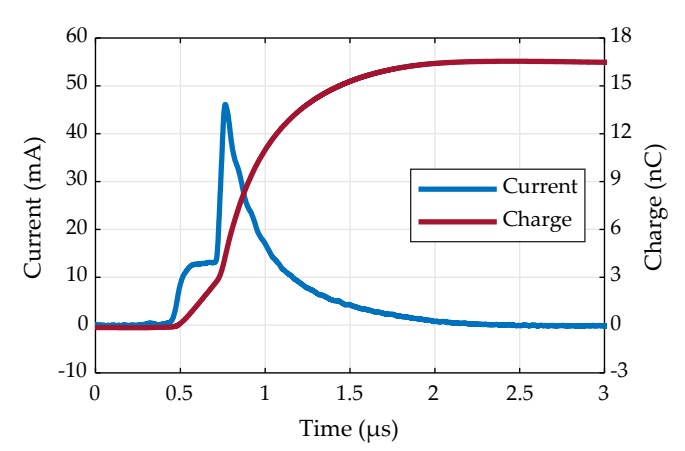

(b)

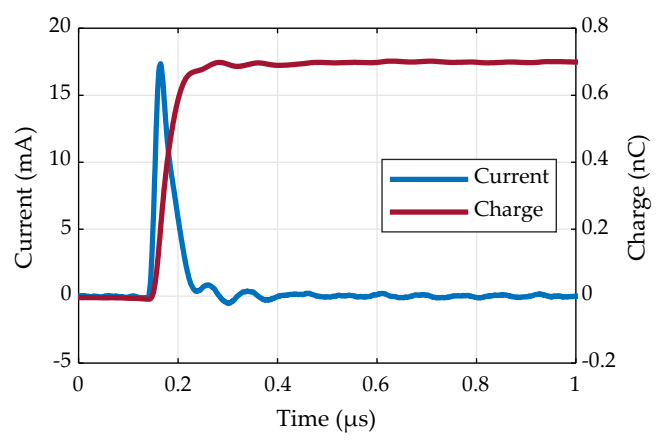

(c)

Figure 10. Positive polarity current pulses and calculated apparent charge for PTFE of $4 \mathrm{~mm}$ thickness in: (a) dry air, (b) nitrogen $\left(\mathrm{N}_{2}\right)$ and (c) carbon dioxide $\left(\mathrm{CO}_{2}\right)$. 
Table 1. Positive polarity current pulses characteristics.

\begin{tabular}{cccccc}
\hline Gaseous Medium & Current Peak (mA) & Rise Time (ns) & Fall Time (ns) & Width (ns) & Duration (ns) \\
\hline Dry air & 37.07 & 28.49 & 263.42 & 110.26 & 326.21 \\
$\mathrm{~N}_{2}$ & 46.14 & 274.25 & 662.93 & 192.94 & 975.02 \\
$\mathrm{CO}_{2}$ & 17.35 & 13.77 & 49.06 & 35.54 & 73.08 \\
\hline
\end{tabular}

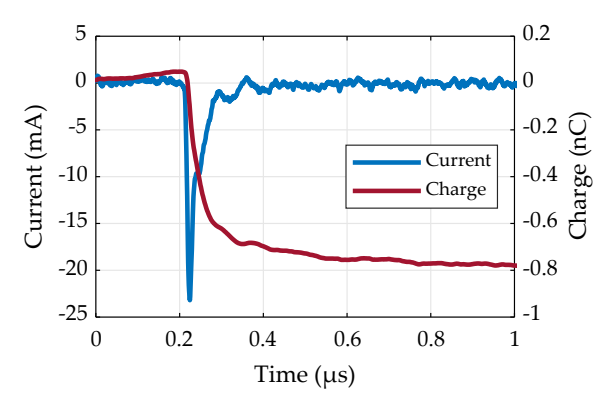

(a)

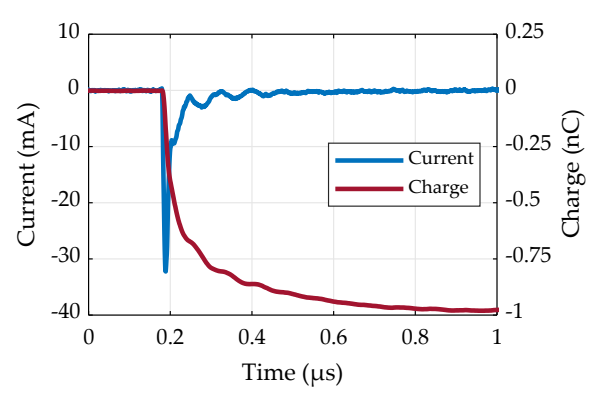

(b)

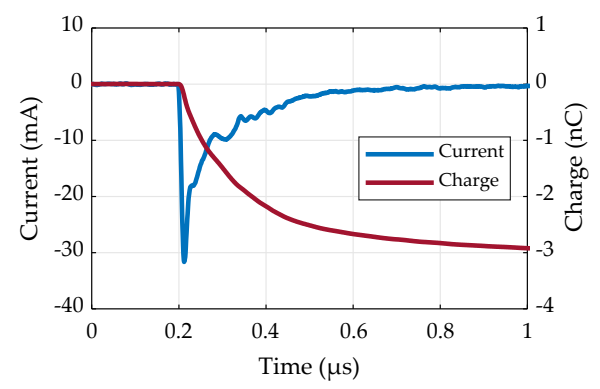

(c)

Figure 11. Negative polarity current pulses and calculated apparent charge for PTFE of $4 \mathrm{~mm}$ thickness in: (a) dry air, (b) nitrogen $\left(\mathrm{N}_{2}\right)$ and (c) carbon dioxide $\left(\mathrm{CO}_{2}\right)$.

Table 2. Negative polarity current pulses characteristics.

\begin{tabular}{cccccc}
\hline Gaseous Medium & Current Peak (mA) & Rise Time (ns) & Fall Time (ns) & Width (ns) & Duration (ns) \\
\hline Dry air & -23.19 & 51.81 & 7.95 & 16.14 & 65.00 \\
$\mathrm{~N}_{2}$ & -32.23 & 41.63 & 5.76 & 12.94 & 52.04 \\
$\mathrm{CO}_{2}$ & -31.63 & 232.38 & 8.53 & 38.58 & 247.65 \\
\hline
\end{tabular}

Significant differences in the measured rise and fall times are observed. For all the tests performed for the purposes of the presented work, the fastest rise and fall times, for these kinds of pulses, were observed in carbon dioxide $\left(\mathrm{CO}_{2}\right)$ while the slowest were seen in nitrogen $\left(\mathrm{N}_{2}\right)$. The calculated apparent charge appears to be dependent and proportional to these values, with the highest charge being observed for the case of $\mathrm{N}_{2}$ in Figure 10b. In [22], the authors describe the correlation between rise and fall times with the electron avalanche process during partial discharge (PD) and electron drift after the full extension of the discharge activity. For the single double-exponential shaped pulses, such an approach can provide valuable information. Figure 11 includes captures of negative polarity current pulses during the negative half cycle of the $\mathrm{AC}$ applied voltage for dry air and $\mathrm{CO}_{2}$, while for nitrogen the negative polarity current pulse appears during the positive AC half cycle. Pulses of opposite polarity to that of the applied voltage were observed in all measurements. Similar findings, using the Pockels effect optical method, were reported in [9] and identified as back-discharges. Following the provided description for the negative discharges during positive polarity of the applied voltage, the negative charge expands uniformly on the positively charged surface neutralizing the positive charges. The opposite process occurs for positive discharges during the negative half cycles. 


\subsection{Current Peaks Density Recordings}

High resolution recordings, within the GS/s sampling rate levels, usually do not allow capturing of multiple trigger events which are able to demonstrate the repeatability of the generated phenomena, during the same applied voltage test. For that purpose, a lower sampling rate, larger buffer size, fast-triggering device was employed. In this way, the quick recording of multiple full AC-cycle trigger events for the same applied voltage attempt was possible without overstressing the test object. Post-processing of these recordings involves isolating the peak values of the current pulses within the time domain and incorporate them in a concatenating plot where colour grading was applied based on the density of these peaks. The colour identification was normalised over the total number of detected peaks based on their polarity. Figures 12-14 illustrate part of the results using the described technique for the cases of $4 \mathrm{~mm}$ thickness insulator samples while Figure 15 includes selected datasets for $6 \mathrm{~mm}$ thick samples. Here, 25 trigger events of $20 \mathrm{~ms}$ duration each are considered, resulting in a total duration of $500 \mathrm{~ms}$ for each case. For all the presented measurements, the applied voltage is approximately equal to the $85 \%$ of the corresponding FOV.

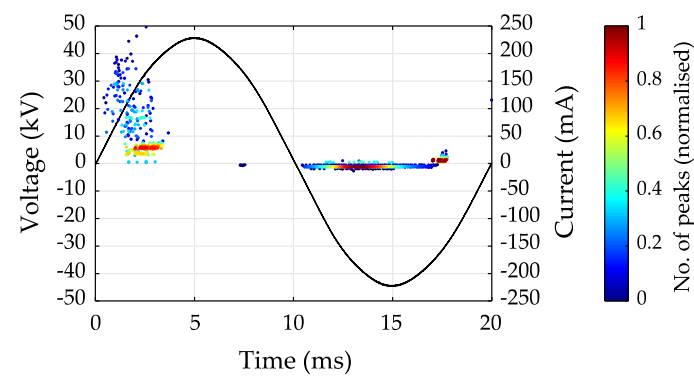

(a)

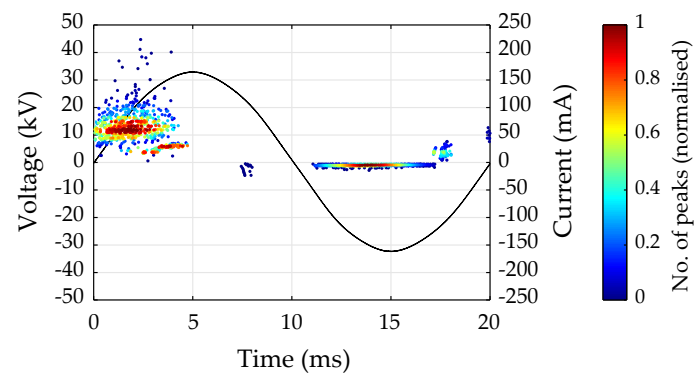

(b)

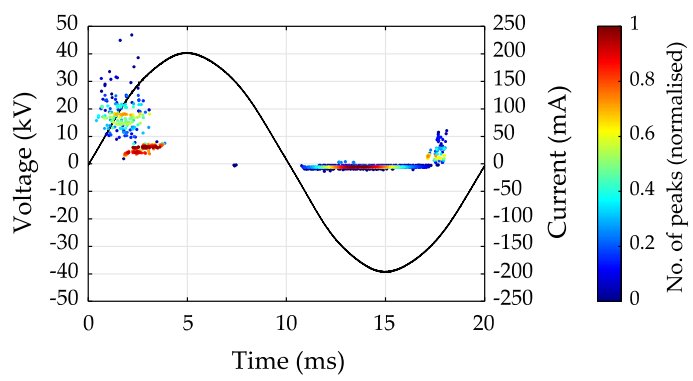

(c)

Figure 12. Current peaks densities for $4 \mathrm{~mm}$ thickness insulator samples in dry air at 1 bar absolute pressure: (a) PTFE, (b) epoxy resin and (c) silicone rubber.

In Figure 12, for samples in dry air, $4 \mathrm{~mm}$ thickness samples of PTFE and silicone rubber have fairly similar behaviour while epoxy resin shows a higher density of discharge activity during the positive half cycle of the applied voltage. For samples of the same thickness in $\mathrm{N}_{2}$ in Figure 13, negative polarity pulses during the negative half cycle were not detected. For silicone rubber, a high density of low amplitude positive polarity discharges was found during the positive half cycles while, for epoxy resin samples, very high positive peaks were captured, possibly indicating that any increase in the applied voltage could have resulted in a flashover. PTFE shows a well distributed activity within the first quarter of the applied voltage waveform. It could be said that $\mathrm{CO}_{2}$ in Figure 14 shows the most stable behaviour. PTFE and silicone rubber once again are very similar while PTFE shows less dense activity during the negative polarity of the applied voltage. For tests with the epoxy resin sample, no detectable pulses were recorded during the second half of the AC waveform for that specific applied voltage level. Tests with thicker samples in Figure 15 show that PTFE in $\mathrm{CO}_{2}$ shows decreased peak amplitudes during the negative half cycle, while epoxy resin in $\mathrm{N}_{2}$ shows considerably lower 
peaks and high repeatability for the $6 \mathrm{~mm}$ samples compared to the $4 \mathrm{~mm}$. Silicone rubber of $6 \mathrm{~mm}$ thickness in dry air shows increased resistance to creeping discharge activity in comparison with the $4 \mathrm{~mm}$ sample of the same material, especially when the polarity of the applied voltage is positive. Overall, the dependence of the insulator sample thickness is visible for the presented cases. For tests with epoxy resin of $4 \mathrm{~mm}$ thickness in $\mathrm{CO}_{2}$, the applied voltage margin for occurrence of the discharge activity in the negative half cycle until flashover is small. Results of $\mathrm{N}_{2}$ combined with $4 \mathrm{~mm}$ thickness samples need further investigation, especially for the case of epoxy resin.

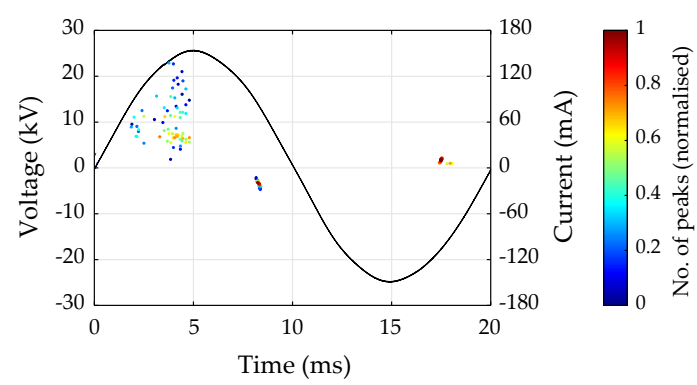

(a)

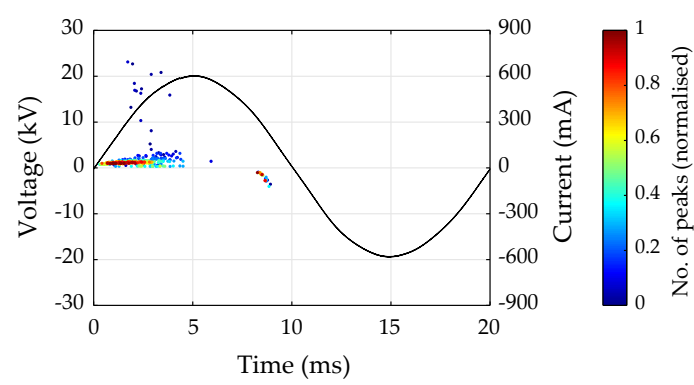

(b)

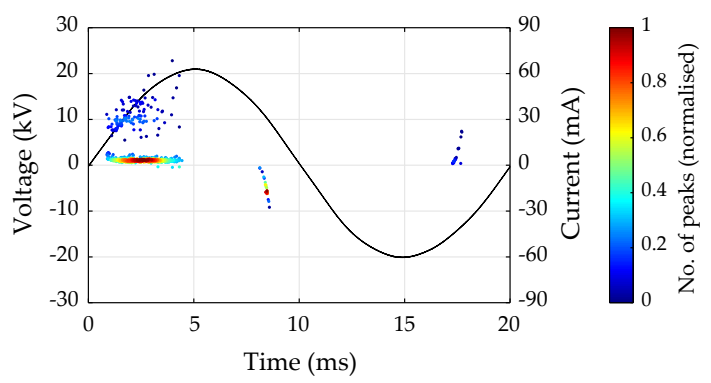

(c)

Figure 13. Current peaks densities for $4 \mathrm{~mm}$ thickness insulator samples in nitrogen $\left(\mathrm{N}_{2}\right)$ at 1 bar absolute pressure: (a) PTFE, (b) epoxy resin and (c) silicone rubber.

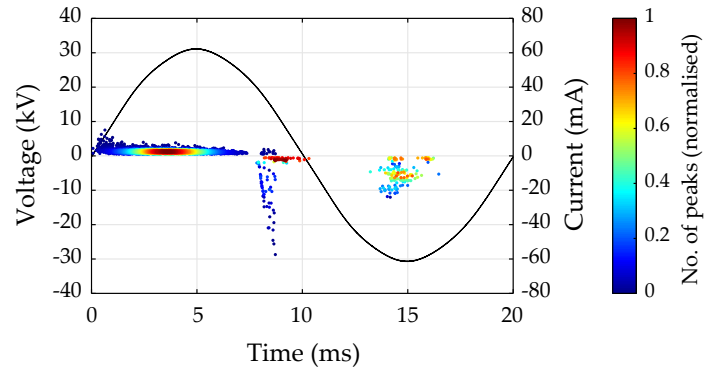

(a)

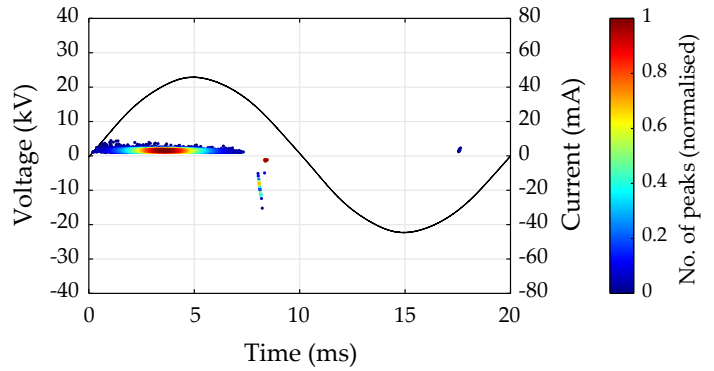

(b)

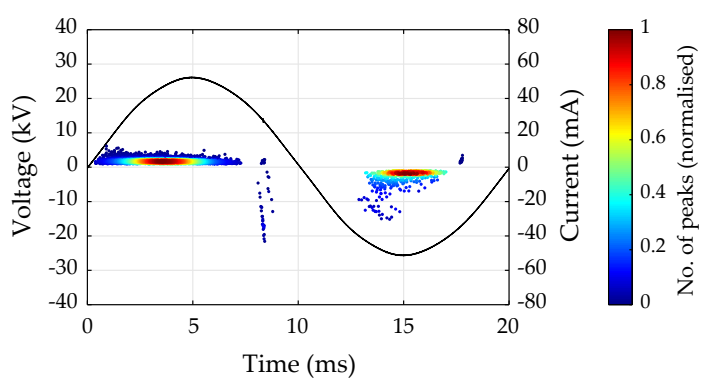

(c)

Figure 14. Current peaks densities for $4 \mathrm{~mm}$ thickness insulator samples in carbon dioxide $\left(\mathrm{CO}_{2}\right)$ at 1 bar absolute pressure: (a) PTFE, (b) epoxy resin and (c) silicone rubber. 


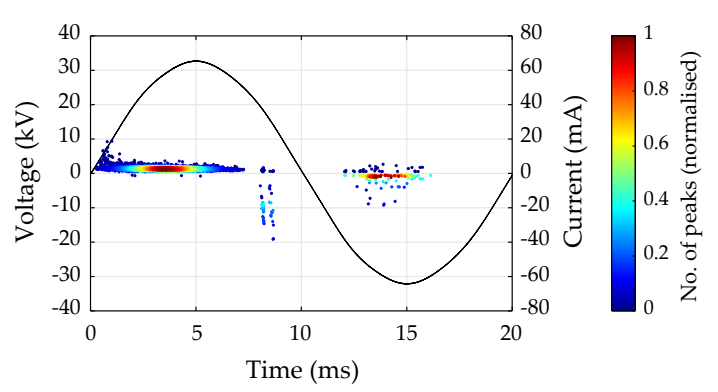

(a)

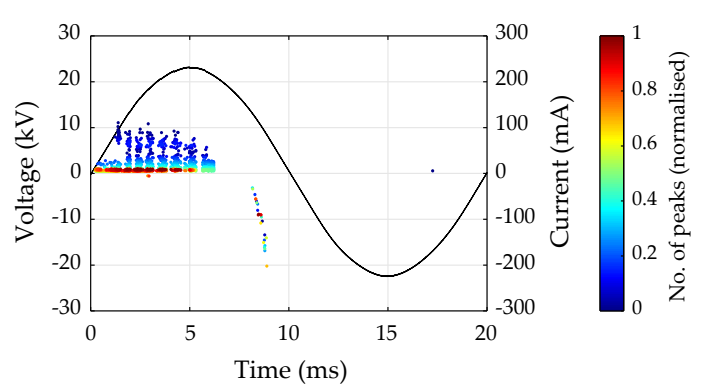

(b)

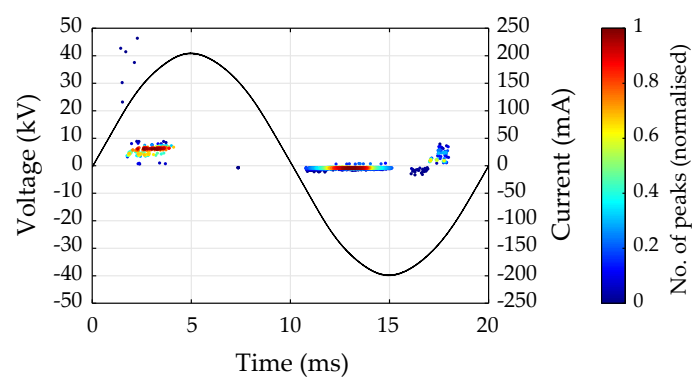

(c)

Figure 15. Current peaks densities for $6 \mathrm{~mm}$ thickness insulator samples: (a) PTFE in $\mathrm{CO}_{2}$ at 1 bar absolute pressure, (b) epoxy resin in $\mathrm{N}_{2}$ at 1 bar absolute pressure and (c) silicone rubber in dry air at 1 bar absolute pressure.

\section{Conclusions}

In this paper, electrical detection of creeping discharge under AC voltage application was presented. The complete procedure, starting from the numerical simulation of the electrode configuration until the final test implementation was described in detail. Emphasis was given to the selection of current sensing and recording devices so that their cut-off frequency is kept as high as possible.

Flashover tests results showed that, for the examined electric field distribution and for all the examined insulating gaseous media (dry air, $\mathrm{CO}_{2}$ and $\mathrm{N}_{2}$ ), lower flashover voltages were measured for electrode configurations with insulating materials having higher relative permittivity. Furthermore, high-frequency recordings of the current pulses associated with creeping discharges clearly indicated that there is more significant electrical discharge activity when the relative permittivity of the insulating material is higher. For lower permittivity insulator sample materials, fewer current pulses were detected however, the activity was spread greatly within a range corresponding to relatively higher amplitudes. It could be said that, the higher the difference between the relative permittivities of the insulator sample material and the insulating gaseous medium, the stronger the detected creeping discharge events are, especially for the cases of dry air and $\mathrm{N}_{2}$. For the case of $\mathrm{CO}_{2}$, the higher the relative permittivity of the insulator material the narrower the margin between creeping discharge and flashover is. Back-discharges were detected for all the cases tested in this investigation.

Future research will extend the current work and further explore the presented scenario using different physical perspectives and detection techniques.

Author Contributions: Conceptualization, M.M., P.W., A.B. and A.H.; methodology, M.M.; software, M.M.; validation, P.W., A.B. and A.H.; formal analysis, M.M.; investigation, M.M.; data curation, M.M.; writing-original draft preparation, M.M.; writing-review and editing, M.M., P.W., A.B. and A.H.; visualization, M.M.; supervision, P.W., A.B. and A.H.

Funding: The initial research leading to this work received funding from the Royal Academy of Engineering through a DVF to Professor A. Beroual to visit Professor Manu Haddad at Cardiff University, "Environmentally-friendly insulating gases for high voltage equipment", Grant number (DVF1617 \5 (28): https://www.raeng.org.uk/grants-and-prizes/grants/international-research-and-collaborations / distinguished-visiting-fellowships. In support to the DVF award, the PhD studentship awarded to Michail Michelarakis was funded by the School of Engineering at Cardiff University. 
Conflicts of Interest: The authors declare no conflict of interest. The funders had no role in the design of the study; in the collection, analyses, or interpretation of data; in the writing of the manuscript, or in the decision to publish the results.

\section{References}

1. Allen, N.L.; Mikropoulos, P.N. Streamer propagation along insulating surfaces. IEEE Trans. Dielectr. Electr. Insul. 1999, 6, 357-362, doi:10.1109/94.775623. [CrossRef]

2. Allen, N.L.; Mikropoulos, P.N. Dynamics of streamer propagation in air. J. Phys. D Appl. Phys. 1999, 32, 913-919, doi:10.1088/0022-3727/32/8/012. [CrossRef]

3. Akyuz, M.; Gao, L.; Cooray, V.; Gustavsson, T.G.; Gubanski, S.M.; Larsson, A. Positive streamer discharges along insulating surfaces. IEEE Trans. Dielectr. Electr. Insul. 2001, 8, 902-910, doi:10.1109/94.971444. [CrossRef]

4. Sadaoui, F.; Beroual, A. DC creeping discharges over insulating surfaces in different gases and mixtures. IEEE Trans. Dielectr. Electr. Insul. 2014, 21, 2088-2094, doi:10.1109/TDEI.2014.004486. [CrossRef]

5. Sadaoui, F.; Beroual, A. AC creeping discharges propagating over solid-gas interfaces. IET Sci. Meas. Technol. 2014, 8, 595-600, doi:10.1049/iet-smt.2014.0050. [CrossRef]

6. Beroual, A.; Coulibaly, M.L.; Aitken, O.; Girodet, A. Investigation on creeping discharges propagating over epoxy resin and glass insulators in the presence of different gases and mixtures. Eur. Phys. J. Appl. Phys. 2011, 56, 30802, doi:10.1051/epjap/2011110122. [CrossRef]

7. Beroual, A.; Khaled, U.; Coulibaly, M.L. Experimental Investigation of the Breakdown Voltage of $\mathrm{CO}_{2}, \mathrm{~N}_{2}$, and $\mathrm{SF}_{6}$ Gases, and $\mathrm{CO}_{2}-\mathrm{SF}_{6}$ and $\mathrm{N}_{2}-\mathrm{SF}_{6}$ Mixtures under Different Voltage Waveforms. Energies 2018, 11, doi:10.3390/en11040902. [CrossRef]

8. Murooka, Y.; Takada, T.; Hiddaka, K. Nanosecond surface discharge and charge density evaluation Part I: review and experiments. IEEE Electr. Insul. Mag. 2001, 17, 6-16, doi:10.1109/57.917527. [CrossRef]

9. Zhu, Y.; Takada, T.; Inoue, Y.; Tu, D. Dynamic observation of needle-plane surface-discharge using the electro-optical Pockels effect. IEEE Trans. Dielectr. Electr. Insul. 1996, 3, 460-468, doi:10.1109/94.506221. [CrossRef]

10. Kumara, S.; Alam, S.; Hoque, I.R.; Serdyuk, Y.V.; Gubanski, S.M. DC flashover characteristics of a polymeric insulator in presence of surface charges. IEEE Trans. Dielectr. Electr. Insul. 2012, 19, 1084-1090, doi:10.1109/TDEI.2012.6215116. [CrossRef]

11. Winter, A.; Kindersberger, J. Surface charge accumulation on insulating plates in $\mathrm{SF}_{6}$ and the effect on $\mathrm{DC}$ and $\mathrm{AC}$ breakdown voltage of electrode arrangements. In Proceedings of the Annual Report Conference on Electrical Insulation and Dielectric Phenomena, Cancun, Mexico, 20-24 October 2002; pp. 757-761.

12. Nakanishi, K.; Yoshioka, A.; Arahata, Y.; Shibuya, Y. Surface Charging On Epoxy Spacer At DC Stress In Compressed $\mathrm{SF}_{6}$ Gas. IEEE Trans. Power Appar. Syst. 1983, PAS-102, 3919-3927, doi:10.1109/TPAS.1983.317931. [CrossRef]

13. Ziegler, S.; Woodward, R.C.; Iu, H.H.; Borle, L.J. Current Sensing Techniques: A Review. IEEE Sens. J. 2009, 9, 354-376, doi:10.1109/JSEN.2009.2013914. [CrossRef]

14. British Standard, B.S. High-Voltage Test Techniques_Part 1: General Definitions and Test Requirements; BSI; BS EN 60060-1:2010; 28-02-2011.

15. Reid, A.J.; Judd, M.D.; Stewart, B.G.; Fouracre, R.A. Partial discharge current pulses in $\mathrm{SF}_{6}$ and the effect of superposition of their radiometric measurement. J. Phys. D Appl. Phys. 2006, 39, 4167-4177, doi:10.1088/0022-3727/39/19/008. [CrossRef]

16. Saitoh, H.; Morita, K.; Kikkawa, T.; Hayakawa, N.; Okubo, H. Impulse partial discharge and breakdown characteristics of rod-plane gaps in $\mathrm{N}_{2} / \mathrm{SF}_{6}$ gas mixtures. IEEE Trans. Dielectr. Electr. Insul. 2002, 9, 544-550, doi:10.1109/TDEI.2002.1024431. [CrossRef]

17. Judd, M.D.; Farish, O. High bandwidth measurement of partial discharge current pulses. In Proceedings of the Conference Record of the 1998 IEEE International Symposium on Electrical Insulation (Cat. No.98CH36239), Washington, DC, USA, 7-10 June 1998.

18. Mansour, D.A.; Kojima, H.; Hayakawa, N.; Hanai, M.; Okubo, H. Physical mechanisms of partial discharges at nitrogen filled delamination in epoxy cast resin power apparatus. IEEE Trans. Dielectr. Electr. Insul. 2013, 20, 454-461, doi:10.1109/TDEI.2013.6508747. [CrossRef] 
19. Rodrigo Mor, A.; Castro Heredia, L.C.; Muñoz, F.A. A Novel Approach for Partial Discharge Measurements on GIS Using HFCT Sensors. Sensors 2018, 18, 4482, doi:10.3390/s18124482. [CrossRef] [PubMed]

20. Zachariades, C.; Shuttleworth, R.; Giussani, R.; MacKinlay, R. Optimization of a High-Frequency Current Transformer Sensor for Partial Discharge Detection Using Finite-Element Analysis. IEEE Sens. J. 2016, 16, 7526-7533, doi:10.1109/JSEN.2016.2600272. [CrossRef]

21. Hu, X.; Siew, W.H.; Judd, M.D.; Peng, X. Transfer function characterization for HFCTs used in partial discharge detection. IEEE Trans. Dielectr. Electr. Insul. 2017, 24, 1088-1096, doi:10.1109/TDEI.2017.006115. [CrossRef]

22. Okubo, H.; Hayakawa, N.; Matsushita, A. The relationship between partial discharge current pulse waveforms and physical mechanisms. IEEE Electr. Insul. Mag. 2002, 18, 38-45, doi:10.1109/MEI.2002.1014966. [CrossRef]

(C) 2019 by the authors. Licensee MDPI, Basel, Switzerland. This article is an open access article distributed under the terms and conditions of the Creative Commons Attribution (CC BY) license (http://creativecommons.org/licenses/by/4.0/). 